\title{
Performance of Ratio of Standardized Regression Slope to Semi- Partial Correlation in Identifying Ill-Conditioned Model
}

\author{
Ijomah Maxwell Azubuike ${ }^{1 *}$, Dozie Kelechi Celestine Nosike ${ }^{2}$ \\ ${ }^{l}$ Department of Mathematics/Statistics, University of Port Harcourt, Choba, Rivers State. \\ ${ }^{2}$ Department of Mathematics/Statistics, Imo-State University, Owerri, Imo-State.
}

*Corresponding Author: Ijomah Maxwell Azubuike, Department of Mathematics/Statistics, University of Port Harcourt, Choba, Rivers State.

\begin{abstract}
Linear regression has gained widespread popularity in literature. However, many applications of linear regression have been in situations in which the model data are collinear or 'ill-conditioned.' Collinearity makes it difficult to ascertain the effect of any single variable, produce biased estimates of coefficients for regressors. Although conducting a multicollinearity diagnosis does not solve nor lead to any specific solution of the problem, realizing its potential impact on findings from regression analysis allows a more careful interpretation of data. In this paper, we examine the performance of ratio of standardized regression slope to semi-partial correlation in an ill-conditioned model with some existing methods of collinearity diagnostics. The result shows that ratio of standardized regression slope to semi-partial correlation seem to be more sensitive to the multicollinearity among predictors in the model than the variance inflation factor and condition number methods.
\end{abstract}

Keywords: muticollinearity; standardized regression; semi-partial correlation; variance inflation factor; condition number.

\section{INTRODUCTION}

One of the main problems when applying some of the classical techniques is the collinearity among the variables used in the models. Collinearity (or multicollinearity or ill-conditioning) occurs when independent variables in a regression are so highly correlated that it becomes difficult or impossible to distinguish their individual eff ects on the dependent variable [1]. It is a problem in key driver analysis because, when two independent variables are highly correlated, it becomes difficult to accurately partial out their individual impact on the dependent variable. Also, there is a sufficient amount of "shared" variance that all predictors soak up together (yielding a significant $\mathrm{R}^{2}$ ) but none of the variables account for a significant portion of unique variance as seen in the non-significant slopes for each variable. This often results in regression coefficients that don't appear to be reasonable. Such collinearity problems can sometimes lead to serious stability problems when the methods are applied [2-3]. While this makes it easy to observe the effects of collinearity in the data, developing a solution may not be straightforward [4].

Multicollinearity among explanatory variables has received a lot of attention in econometric theory and in econometric texts [6-8]. Hair et al. [9] noted that as multicollinearity increases, it is more difficult to ascertain the effect of any single variable produce biased estimates of coefficients for regressors because the variables have more interrelationships. The literature on linear models with special focus on multicollinearity spans several decades already and provides numerous suggestions for diagnosing the presence of substantive collinearity ranging from simple rules of thumb to complex indices. However, there is still a continuing active interest on the problem.

Although conducting a multicollinearity diagnosis does not solve nor lead to any specific solution of the problem, realizing its potential impact on findings from regression analysis allows a more careful interpretation of data. The literature provides numerous suggestions, ranging from simple rules of thumb to complex indices, for diagnosing the presence of substantive collinearity. [5] also proposed a procedure for detecting multicollinearity which comprised of three tests (i.e. Chi-square test, F-test and T-test). However, these tests have been greatly criticized. [10] claims that the third test, where the authors use the partial-correlation coefficients is ineffective. The use of condition index for collinearity 
diagnostics alone is not enough. Some other insight is necessary to find which columns of $\mathrm{X}$ are involved in the collinearity. [11] has it that multicollinearity is said to be "harmful" if $r_{i j} \geq R^{2}$. Such simple correlation coefficients are sufficient but not necessary condition for multicollinearity. In many cases there are linear dependencies, which involve more than two explanatory variables, that this method cannot detect $[12,13]$ pointed out that using correlation matrix is unable to reveal the presence or number of several coexisting collinear relations [13]. Furthermore, in using variance inflation factor (VIF) some authors have stated that multicollinearity is problematic if largest VIF exceeds value of 10, or if the mean VIF is much greater than 1. Although VIF greater than 5 or VIF greater than 10. [14] are suggested for detecting multicollinearity, there is no universal agreement as what the cut-off based on values of VIF should be used to detect multicollinearity. Caution for misdiagnosis of multicollinearity using low pairwise correlation and low VIF was reported in the literature for collinearity diagnostic as well. [15] demonstrated that VIF rules of thumb should be interpreted with cautions and should be put in context of the effects of other factors that influence the stability of the specific regression coefficient estimate and suggested that any VIF cut-off value should be based on practical consideration. [16] further suggested VIF to be evaluated against the overall fit of the model, using the model $\mathrm{R}^{2}$ statistics. VIF $>1 /\left(1\right.$-overall model $\left.\mathrm{R}^{2}\right)$ indicates that correlation between the predictors is stronger than the regression relationship and multicollinearity can affect their coefficient estimates, while Hair et al.[17] suggest variance inflation factors (VIF) less than 10 are indicative of inconsequential collinearity. However, there is no formal criteria for determining the magnitude of variance inflation factors that cause poorly estimated coefficients. The decision to consider a VIF to be large was essentially arbitrary. In summary, reviewing the literature on ways to diagnosing collinearity reveals several points. First, a variety of alternatives are available and may lead to dramatically different conclusions based on their cutoff points. Second, what might be gained from the different alternatives in any specific empirical situation is often unclear. Part of this ambiguity is likely to be due to inadequate knowledge about what degree of collinearity is "harmful" [18]. In much of the empirical research on collinearity diagnostics, data with extreme levels of collinearity are used to provide rigorous tests of the approach being proposed. Such extreme collinearity is rarely found in actual cross-sectional data. Based on the above premises, the purpose of this paper is to investigate the performance of ratio of standardized regression slope to semi-partial correlation in a collinear data set. To compare the performance of this robust multicollinearity diagnostic method with other existing diagnostic methods (i.e. VIF and Condition number) using a Monte Carlo simulation study. Diagnostics are calculated for a hypothetical regression model with the aim of identifying the degree of collinearity and the variables that are involved (or not involved) in a strong collinear relationship.

The plan of the paper is as follows. In Section 2, the relationship between standardized regression slope to semi partial correlation index with VIF is considered in this section. The simulation and results are presented in Section 3. In the last Section 4, findings and some conclusions are reported.

\section{STANDARDIZED REGRESSION SLOPES}

It is well-known that the standardized regression coefficient in a bivariate regression model is the same as the bivariate correlation coefficient between the independent and dependent variables. In multiple regression analysis, standardized regression coefficients are scale free estimates and are related to correlation coefficients, but the relationship is much more complex than in bivariate regression. The standardized regression slope is considered the most common measure of relative importance in multiple regression analysis. [19] proposed the standardized regression coefficient as an index for synthesizing studies reporting multiple regression analyses. The standardized regression coefficient represents the effect of the focal predictor $(\mathrm{Xj})$ or the criterion variable $(\mathrm{Y})$ while controlling for other predictors in a model in standard deviation units. Although the variation of predictors (Xs) across studies is still a concern, the regression coefficients are standardized, facilitating comparisons across studies.

Standardized coefficients are coefficients adjusted so that they may be interpreted as having the same, standardized scale and the magnitude of the coefficients can be directly compared (ranked)The greater the absolute value of the standardized coefficient, the greater the predicted change in the proba bility of the outcome given a 1standard deviation change in the corresponding predictor variable, hold ing constant the other predictors in the model. For example, consider a regression model with two independent variables,

$$
y_{i}=\beta_{0}+\beta_{1} x_{1 i}+\beta_{2} x_{2 i}+\varepsilon_{i}
$$


where $\mathrm{yi}$ is the score on the dependent variable of the $\mathrm{i}^{\text {th }}$ subject, $\mathrm{x}_{1 \mathrm{i}}$ and $\mathrm{x}_{2 \mathrm{i}}$ are the values of the independent variables for the $\mathrm{i}^{\text {th }}$ subject, $\beta_{0}, \beta_{1}$ and $\beta_{2}$ are population regression coefficients, and $\varepsilon_{i}$ is a residual term, often assumed to be normally distributed with mean of zero and constant variance. The associated standardized regression model is

$$
y_{i}^{*}=\beta_{1}^{*} z_{1}+\beta_{2}^{*} z_{2}+\varepsilon_{i}^{*}
$$

where $\beta_{1}^{*}$ and $\beta_{2}^{*}$ are the standardized regression coefficients in population. The error term, $\varepsilon_{i}^{*}$, is assumed to be normally distributed with mean of zero and variance of $\sigma^{* 2}$.

The following standardization converts the matrix $\mathrm{X}^{\mathrm{T}} \mathrm{X}$ into a correlation matrix.

Let $\mathrm{y}^{*}:=\mathrm{y}^{\mathrm{c}}$ denote the centered dependent variable as before and put

$$
Y_{i}^{*}=\beta_{0}+\beta_{1} Z_{i 1}+\beta_{2} Z_{i 2}+\ldots \ldots \ldots \ldots \ldots \ldots+\beta_{k} Z_{i k}+\varepsilon_{i}
$$

where $Z_{i j}=\frac{X_{i, j}-\bar{X}_{j}}{\sqrt{S_{j j}}} \quad$ and $S_{j j}=\sum_{i=1}^{n}\left(X_{i, j}-\bar{X}_{j}\right)^{2}$

This leads to the standardized model

$$
\frac{Y_{i}-\bar{Y}}{S_{y}}=\hat{\beta}_{1} \frac{\left(X_{i 1}-\bar{X}_{1}\right)}{S_{1}}+\hat{\beta}_{2} \frac{\left(X_{i 2}-\bar{X}_{2}\right)}{S_{2}}+\ldots \ldots+\hat{\beta}_{k} \frac{\left(X_{i k}-\bar{X}_{k}\right)}{S_{k}}+\varepsilon_{i}
$$

putting (4) in vector notation, we have

$$
Y^{*}=Z \beta+\varepsilon^{*}
$$

where $\quad Y^{*}=P Y, \beta=\mathrm{A} \alpha_{\left(\alpha_{0}\right)}, \varepsilon^{*}=P \varepsilon \quad$ and $\quad Z=\left[Z_{1}, Z_{2}, \ldots \ldots, Z_{k}\right]=P X_{(1)} \mathrm{A}^{-1}$

$$
\mathrm{A}=\left[\begin{array}{lll}
\sqrt{S_{11}} & & \\
& . . & \\
& & \sqrt{S_{k k}}
\end{array}\right]
$$

All of the scaled regressors have sample mean equal to zero and the Euclidean norm of each column $Z_{j}:=[Z]_{1 \leq i \leq n}, j=1, \ldots, k$ of $Z$ is equal to one. The least squares estimator of (5) is given by

$\hat{\beta}=\left(Z^{T} Z\right)^{-1} Z^{T} Y^{*}$

$$
\begin{aligned}
& =\left(\mathrm{A}^{-1} X_{\{1\}} P X_{\{1\}} \mathrm{A}^{-1}\right)^{-1} \mathrm{~A}^{-1} X^{T}{ }_{\{1\}} P Y \\
& =\mathrm{A}\left(X_{\{1\}} P X_{\{1\}}\right)^{-1} X^{T}{ }_{\{1\}} P Y=\mathrm{A} \hat{\alpha}_{\left(\alpha_{0}\right)}
\end{aligned}
$$

The coefficients for the standardized model is given by

$$
\hat{\alpha}_{j}=\alpha_{j}\left(\frac{S_{j}}{S_{y}}\right) j=1,2, \ldots \ldots . ., k
$$

and thus the relationship between the estimates of the original and standardized regression coefficients is given by

$$
\hat{\alpha}_{j j}=\beta_{j}\left(\frac{1}{S_{y}}\right)^{\frac{1}{2}} \quad j=1,2, \ldots \ldots . ., k
$$

The least squares estimates for a trivariate standardized regression is

$$
\begin{aligned}
& \hat{Z}_{y}=\beta_{1}^{*} Z_{1}+\beta_{2}^{*} Z_{2} \\
& \beta_{1}^{*}=\frac{r_{y 1}-r_{12} r_{y 2}}{1-r_{12}^{2}} \text { and } \beta_{2}^{*}=\frac{r_{y 2}-r_{12} r_{y 1}}{1-r_{12}^{2}}
\end{aligned}
$$


where $\mathrm{r}_{\mathrm{y} 1}$ is the simple correlation coefficient between $\mathrm{y}$ and $\mathrm{x}_{1}, \mathrm{r}_{\mathrm{y} 2}$ is the simple correlation coefficient between $\mathrm{y}$ and $\mathrm{x}_{2}$, and $\mathrm{r}_{12}$ is the simple correlation coefficient between $\mathrm{x}_{1}$ and $\mathrm{x}_{2}$. The standardized regression coefficient for the first independent variable, $b_{1}^{*}$, is a function of all the correlation coefficients among the variables. When the intercorrelation between the two independent variables is zero (i.e., $\mathrm{r}_{12}=0$ ), the standardized regression coefficient, $b_{1}^{*}$, is equal to the correlation coefficient, $\mathrm{y}_{1 \mathrm{r}}$. For multiple regression models with more predictors, these formulas are more complex, but the simplification that $b_{1}^{*}=r_{y 1}$ holds if all the intercorrelation values among the predictors are zero Peterson and Brown [20] investigated the empirical relationship between simple correlation coefficients and standardized regression slopes. In this study 1,504 standardized regression coefficients and correlation coefficients from published articles in behavioral journals were collected. The authors provided the estimated slope of the regression of the standardized regression slope on the simple correlation coefficient, and found a strong relation between simple correlation coefficients and standardized regression slopes.

\section{Semi-Partial Correlation Index}

Aloe [21] proposed the semi-partial correlation index for synthesizing slopes in multiple regression models. The semi-partial correlation can typically be obtained from studies reporting multiple regressions. Aloe and Becker [22] proposed using the semi-partial correlation as a partial effect size in meta-analysis. Specifically, the semi-partial correlation can also be computed as follows:

$$
r_{s p}=t \sqrt{\frac{1-R^{2}}{n-p-1}}
$$

where $\mathrm{t}$ is the $\mathrm{t}$-statistic for the focal predictor, $\mathrm{R}^{2}$ is the variance explained by the model, $\mathrm{n}$ is the total sample size, and $\mathrm{p}$ is the number of predictors. The semi-partial correlation index represents the unique effect of the focal predictor on the target outcome partialling out the effects of other predictors in the model. This index does not include the common effect shared with other predictors on the outcome. Thus, when the number of predictors is increased, the values of the semi-partial correlation index tend to be smaller. A squared semi-partial correlation represents the proportion of all the variance in $\mathrm{Y}$ that is associated with one predictor but not with any of the other predictor. Squared Semi-partial correlation indicates variable importance because it measures incremental value in R-Square. We can rank variables based on high to low values of squared semi-partial correlation score. In terms of residuals, the semipartial correlation for $\mathrm{X}_{\mathrm{i}}$ is the $\mathrm{r}$ between all of $\mathrm{Y}$ and $\mathrm{X}_{\mathrm{i}}$ from which the effects of all other predictors have been removed.

\section{Relationship with Variance Inflation Factor}

The semi-partial correlation coefficient which is obtained by

$$
r_{s p}=\frac{r_{Y 1}-r_{12} r_{Y 2}}{\sqrt{1-r_{12}^{2}}}
$$

Now we consider the ratio of the standardized regression slope to the semi-partial correlation given by

$$
\frac{\beta *}{r_{s p}}=\frac{\frac{r_{Y 1}-r_{12} r_{Y 2}}{1-r_{12}^{2}}}{\frac{r_{Y 1}-r_{12} r_{Y 2}}{\sqrt{1-r_{12}^{2}}}}=\frac{1}{\sqrt{1-r_{12}^{2}}}=\sqrt{\frac{1}{1-r_{12}^{2}}}=\sqrt{V I F}
$$

Noting that $V I F=\frac{1}{1-r_{12}^{2}}$

In summary, the standardized regression slope is related to the raw slope and the semi partial correlation. The relation among these three values can be written as

$$
\beta^{*}=\beta \frac{S_{X}}{S_{Y}}=r_{s p} \sqrt{V I F},
$$

and the relations among the standard errors of these estimates can be written as 
Performance of Ratio of Standardized Regression Slope to Semi-Partial Correlation in Identifying IllConditioned Model

$$
S E\left(\beta^{*}\right)=S E(\beta) \frac{S_{X}}{S_{Y}}=S E\left(r_{s p}\right) \sqrt{V I F} \text { under the large sample condition. }
$$

\section{Materials ANd Methods}

In this section, the estimation of standardized regression coefficients in the cases of three predictor in a collinear regression models are addressed. The ordinary least squares estimators of the standardized regression parameters to semi partial correlation are presented and compared with other existing methods of detecting presence of multicollinearity. In addition, a method is presented for obtaining the standard errors of standardized regression slopes. An investigation of the difference between the standardized regression slope in a two-predictor model and the simple correlation coefficient is presented. Finally, we compare the standardized regression slope to the semi-partial correlation with VIF and condition index while varying the number of predictors and intercorrelations among predictors.

\section{Simulation}

To understand the role of different diagnostic techniques more clearly we require simulation results with a good number of replications considering a variety of sample sizes with three normally distributed variables $\left(X_{1}-X_{3}\right)$. We have used the design (9) with $\mathrm{n}=50,100,200,500,1000$ and 10000 and the results of intercorrelation for different estimation techniques and different sample sizes using SAS version 9 software. The variables were created in such a way that $X_{1}, X_{2}$ and $X_{3}$ are highly correlated by perturbing the error U=RANUNI (START) with $2,4,6,8$, and 10 . All of the explanatory variables were fixed at 0.5 in value, with mean values of zero and standard deviations of one. This method was chosen because it is commonly used and well-understood.

$\mathrm{U}=$ ranuni (start)

$\mathrm{X}_{1}=\mathrm{U}+$ rannor (start) $* 0.5$

$\mathrm{X}_{2}=\mathrm{U}+$ rannor (start) $* 0.5$

$\mathrm{X}_{3}=\mathrm{U}+$ rannor (start) $* 0.5$

$\mathrm{Y}=1+\mathrm{X}_{1}+\mathrm{X}_{2}+$ rannor (start)

That is, to achieve the objective of varying collinearity, we perturb the random error $\mu$ by multiplying it with values (i.e. 2, 4, 6, 8, and 10). This generates different values of the correlation coefficient among the three explanatory variables as shown in table.

\section{RESUlt OF ANALYSIS}

Table 1 below shows the comparison of the standardized regression slope and the semi-partial correlation while varying the number of predictors and intercorrelations. The first column in Table 1 represents the sample size, the second column is the intercorrelation among the explanatory variables $\left(\rho_{\mathrm{xy}}\right)$, the third column is the standardized regression slope $\left(\beta^{*}\right)$, the fourth column is the semi-partial correlation coefficient $\left(\mathrm{r}_{\mathrm{sp}}\right)$, the fifth column shows the difference between the standardized regression slope and the semi-partial correlation coefficient $\left(\beta^{*}-r_{\mathrm{sp}}\right)$, the sixth column represents the ratio of the standardized regression slope to semi-partial correlation coefficient, the next column represents the variance inflation factor (VIF) and the last column is the condition number $(\mathrm{CN})$.

Table1. Comparison of the Standardized Regression Slope and the Semi-partial Correlation with Variance Inflation Factor and Condition Number

\begin{tabular}{|c|c|c|c|c|c|c|c|c|}
\hline $\mathrm{n}$ & $\rho_{x y}$ & STD ERROR & $\beta^{*}$ & $r_{s p}$ & $\beta^{*}-r_{s p}$ & $\frac{\beta^{*}}{r_{s p}}$ & $V I F$ & $C N$ \\
\hline 50 & \multirow{6}{*}{0.30} & 0.22861 & 0.43398 & 0.359207 & 0.074773 & 1.208160 & 1.45963 & 27.76 \\
\hline 100 & & 0.92639 & 0.40338 & 0.333392 & 0.069988 & 1.209928 & 1.46402 & 21.87 \\
\hline 200 & & 0.97960 & 0.44125 & 0.344427 & 0.096823 & 1.281113 & 1.64127 & 23.30 \\
\hline 500 & & 0.08326 & 0.47061 & 0.359096 & 0.111514 & 1.310541 & 1.71751 & 23.20 \\
\hline 1000 & & 0.05817 & 0.43756 & 0.340367 & 0.097193 & 1.285552 & 1.65266 & 21.21 \\
\hline 10000 & & 0.01746 & 0.45358 & 0.346684 & 0.106896 & 1.308337 & 1.71172 & 21.91 \\
\hline 50 & \multirow{4}{*}{0.70} & 0.21609 & 0.45013 & 0.290345 & 0.159785 & 1.550330 & 2.40357 & 59.38 \\
\hline 100 & & 0.19048 & 0.43808 & 0.28233 & 0.15575 & 1.551662 & 2.40771 & 45.60 \\
\hline 200 & & 0.13330 & 0.47478 & 0.287715 & 0.187065 & 1.650174 & 2.72306 & 49.69 \\
\hline 500 & & 0.08128 & 0.49583 & 0.294669 & 0.201161 & 1.682666 & 2.83149 & 49.30 \\
\hline
\end{tabular}


Performance of Ratio of Standardized Regression Slope to Semi-Partial Correlation in Identifying IllConditioned Model

\begin{tabular}{|c|c|c|c|c|c|c|c|c|}
\hline 1000 & & 0.05659 & 0.46086 & 0.279732 & 0.181128 & 1.647505 & 2.71420 & 45.18 \\
\hline 10000 & & 0.01706 & \begin{tabular}{|l|}
0.47251 \\
\end{tabular} & 0.282046 & 0.190464 & 1.675294 & 2.80665 & 47.34 \\
\hline 50 & \multirow{6}{*}{0.89} & 0.20859 & \begin{tabular}{|l|l|}
0.46390 \\
\end{tabular} & 0.195959 & 0.267941 & 2.367330 & 5.60388 & 160.64 \\
\hline 100 & & 0.18344 & \begin{tabular}{|l|}
0.47010 \\
\end{tabular} & 0.198494 & 0.271606 & 2.368330 & 5.60951 & 122.01 \\
\hline 200 & & \begin{tabular}{|l|}
0.12991 \\
\end{tabular} & \begin{tabular}{|l|}
0.50333 \\
\end{tabular} & 0.200175 & 0.303155 & 2.514451 & 6.32216 & 134.75 \\
\hline 500 & & 0.08001 & \begin{tabular}{|l|l|}
0.51853 \\
\end{tabular} & 0.203715 & 0.314815 & 2.545364 & 6.47848 & 132.35 \\
\hline 1000 & & 0.05554 & 0.48242 & 0.193598 & 0.288822 & 2.491871 & 6.20997 & 122.04 \\
\hline 10000 & & \begin{tabular}{|l|}
0.01680 \\
\end{tabular} & \begin{tabular}{|l|}
0.48961 \\
\end{tabular} & 0.193985 & 0.295625 & 2.523964 & 6.37116 & 128.77 \\
\hline 50 & \multirow{6}{*}{0.93} & 0.20640 & \begin{tabular}{|l|l|}
0.46846 \\
\end{tabular} & 0.144603 & 0.323857 & 3.239631 & 10.49757 & 312.29 \\
\hline 100 & & 0.18092 & \begin{tabular}{|l|}
0.48157 \\
\end{tabular} & 0.148526 & 0.333044 & 3.242326 & 10.51049 & 236.21 \\
\hline 200 & & \begin{tabular}{|l|}
0.12875 \\
\end{tabular} & \begin{tabular}{|l|}
0.51294 \\
\end{tabular} & 0.149332 & 0.363608 & 3.434900 & 11.79617 & 262.47 \\
\hline 500 & & 0.07960 & 0.52655 & 0.152086 & 0.374464 & 3.462197 & 11.98580 & 256.54 \\
\hline 1000 & & 0.05520 & \begin{tabular}{|l|l|}
0.49064 \\
\end{tabular} & 0.144672 & 0.345968 & 3.391395 & 11.50085 & 237.35 \\
\hline 10000 & & 0.01673 & \begin{tabular}{|l|}
0.49609 \\
\end{tabular} & 0.14481 & 0.35128 & 3.425794 & 11.73688 & 250.92 \\
\hline 50 & \multirow{6}{*}{0.97} & 0.20522 & \begin{tabular}{|l|}
0.47039 \\
\end{tabular} & 0.113842 & 0.356548 & 4.131955 & 17.07287 & 514.28 \\
\hline 100 & & 0.17928 & \begin{tabular}{|l|l|}
0.48671 \\
\end{tabular} & 0.117686 & 0.369024 & 4.135665 & 17.09881 & 388.18 \\
\hline 200 & & 0.12802 & \begin{tabular}{|l|}
0.51701 \\
\end{tabular} & 0.118195 & 0.398815 & 4.374221 & 19.13606 & 432.74 \\
\hline 500 & & 0.07936 & \begin{tabular}{|l|}
0.53000 \\
\end{tabular} & 0.120499 & 0.409501 & 4.398378 & 19.34620 & 421.79 \\
\hline 1000 & & 0.05499 & \begin{tabular}{|l|}
0.49449 \\
\end{tabular} & 0.114717 & 0.379773 & 4.310519 & 18.57875 & 391.11 \\
\hline 10000 & & 0.01668 & \begin{tabular}{|l|}
0.49910 \\
\end{tabular} & 0.114804 & 0.384296 & 4.347403 & 18.89659 & 413.75 \\
\hline
\end{tabular}

We observe from the results presented in the above table that the performance of the ratio of standardized regression slope to semi-partial correlation coefficient (RSSR) is very much similar to the variance inflation (VIF) method in the detecting collinearity. But the performance of standardized regression slope to semi-partial correlation coefficient is outstanding. Values obtained by this method are very close to their corresponding errors which are reflected in their very interrelationship. A closer look at the table shows that the ratio of standardized regression slope to semi-partial correlation reveals responds more even to the standard error than variance inflation factor (VIF) and condition number $(\mathrm{CN})$. This is confirmed in the plot of standard error against the selected diagnostic techniques. Figure 1 plots shows the empirical distributions of the standardized regression slopes to semi partial correlation coefficients (RSSC) with the variance inflation factor (VIF) and condition number (CN). As can be seen in Figure 1.1, Figure 1.2 and Figure 1.3 the graph of ratio of standardized regression slope to semipartial correlation responds to every shock in standard error resulting from collinearity. This was unlike the case of variance of inflation factor which responded at the initial stage. The worst case scenario is the condition number which did not respond to the standard error in spite degree of collinearity and sample size.

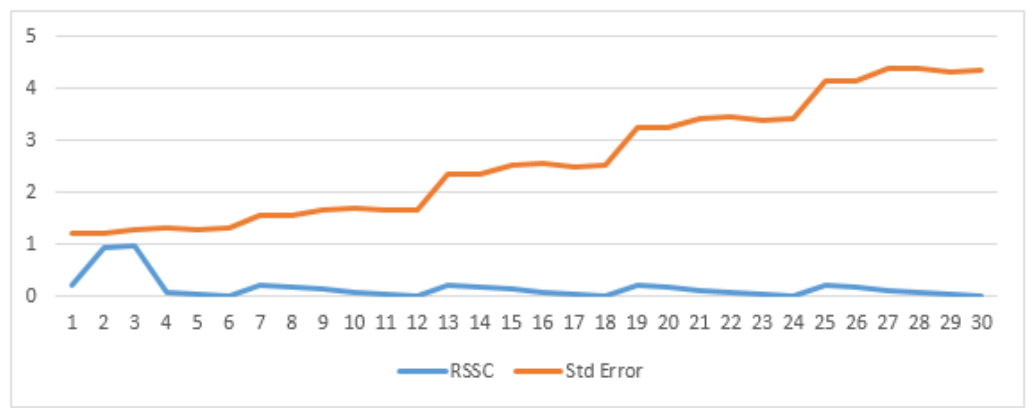

Fig.1.1. Ratio of Standardized Regression slope to semi-partial coefficient with standard error of the model

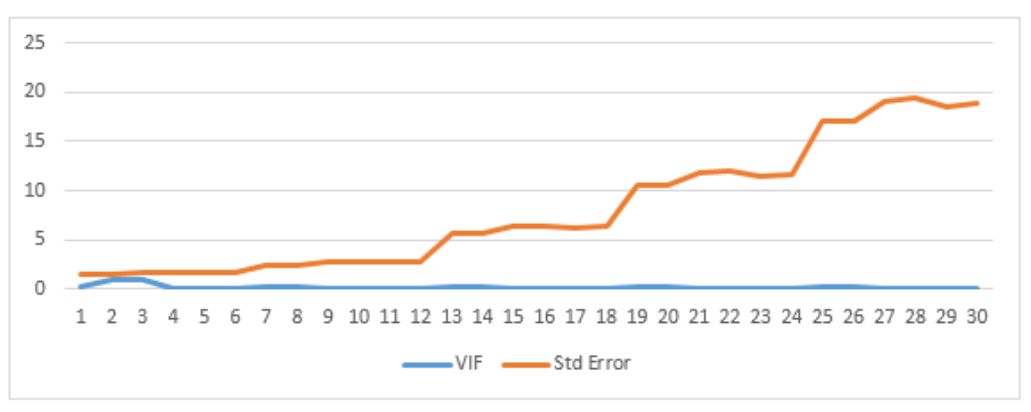

Fig.1.2. Variance Inflation Factor with standard error of the model 
Performance of Ratio of Standardized Regression Slope to Semi-Partial Correlation in Identifying IllConditioned Model

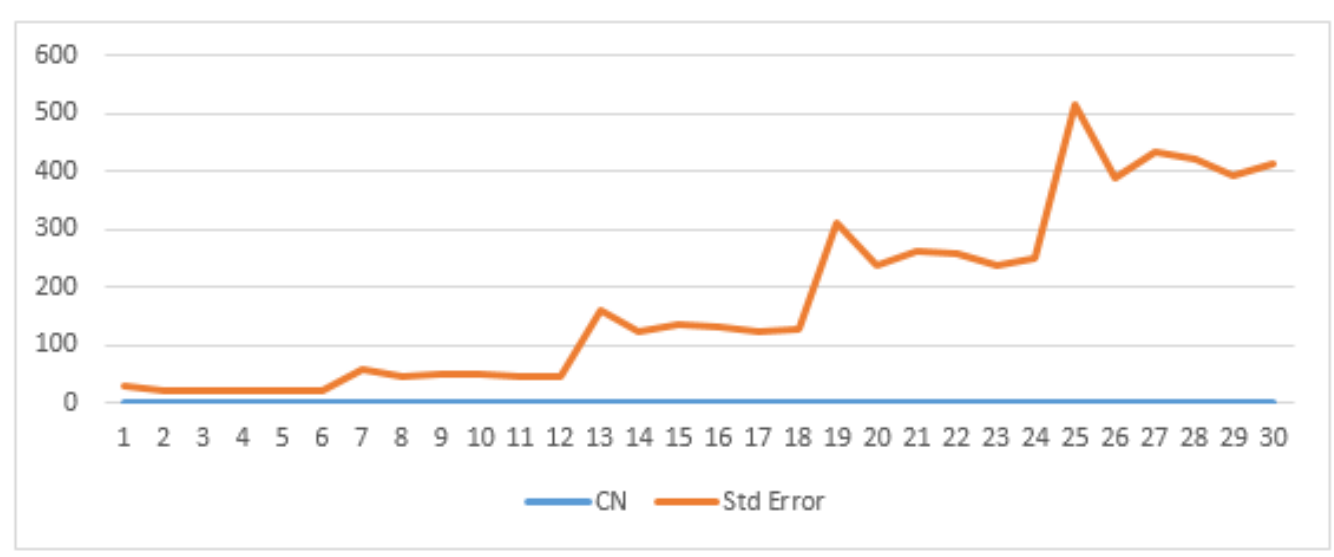

Fig.1.3. Graph of Condition number with standard error of the model

Figure 2 shows the ratio of standardized regression slopes to semi-partial correlation, variance inflation factor and condition number for the three predictor models for all conditions. The graph reveals that the empirical distributions of RSSR become narrower as sample sizes become larger. As shown in Figure 2.1, the semi-partial correlation coefficient dramatically decreases across higher intercorrelations for all panels. The graph shows that the ratio of the standardized slope to semi partial correlation (RSSR) appears to be consistent when compared with VIF which exhibited slight deviation for very strong collinearity as the sample increases. The VIF and $\mathrm{CN}$, the estimated variance inflation factor and condition number have larger variance when predictors are highly correlated for the three predictor models. For condition number, it was trending all through samples. In other words, it is unbounded.

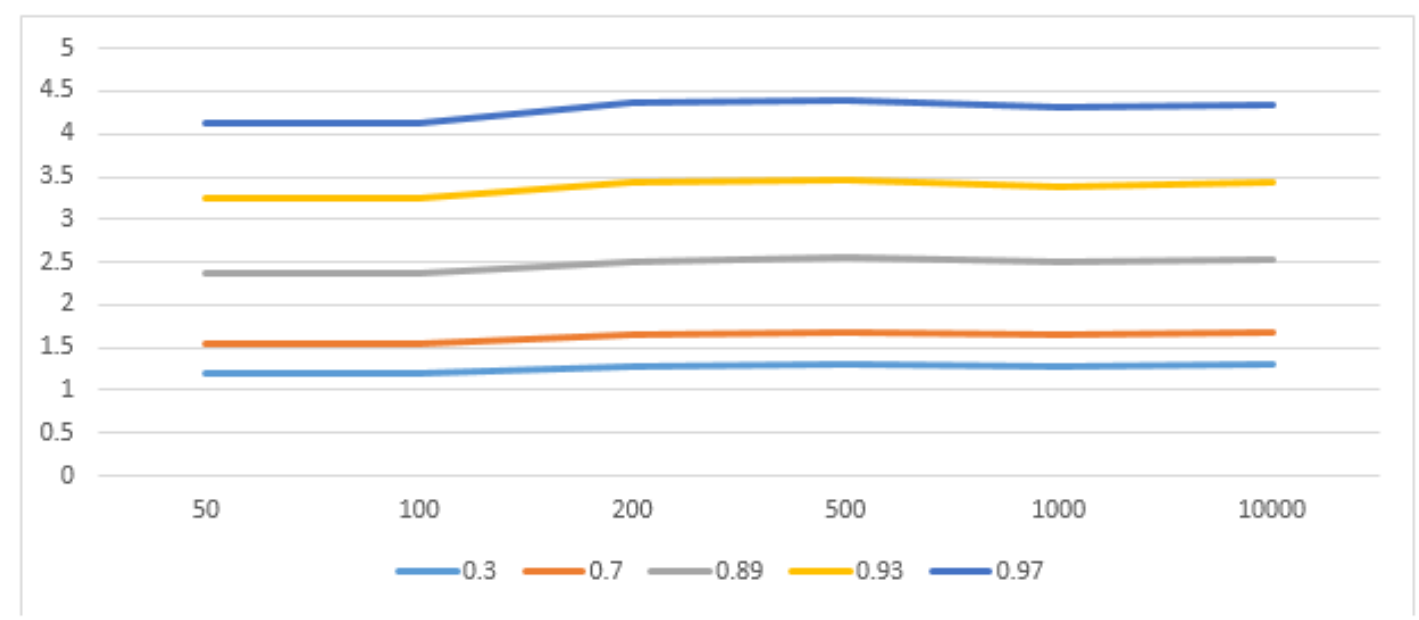

Figure2.1. Ratio of Standardized Regression slope to Semi-partial correlation at different degree of correlation and sample size.

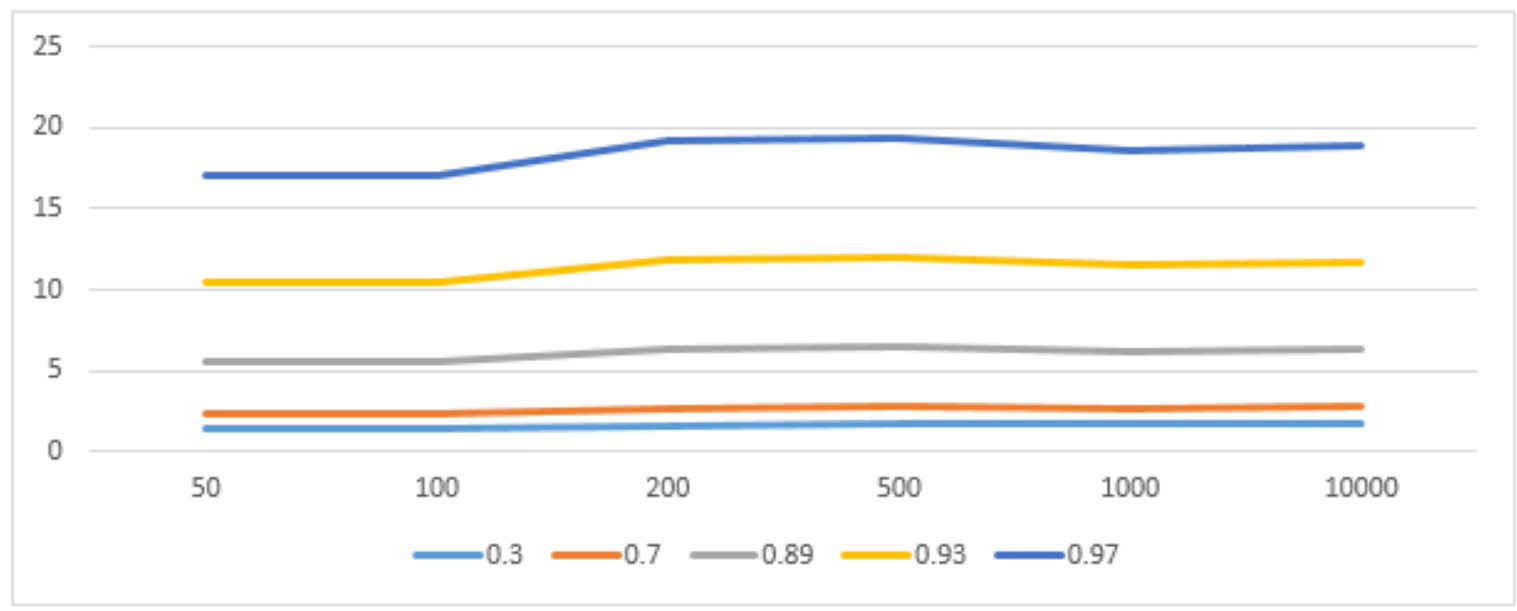

Figure2.2. Graph of Variance Inflation at different degree of correlation and sample size. 
Performance of Ratio of Standardized Regression Slope to Semi-Partial Correlation in Identifying IllConditioned Model

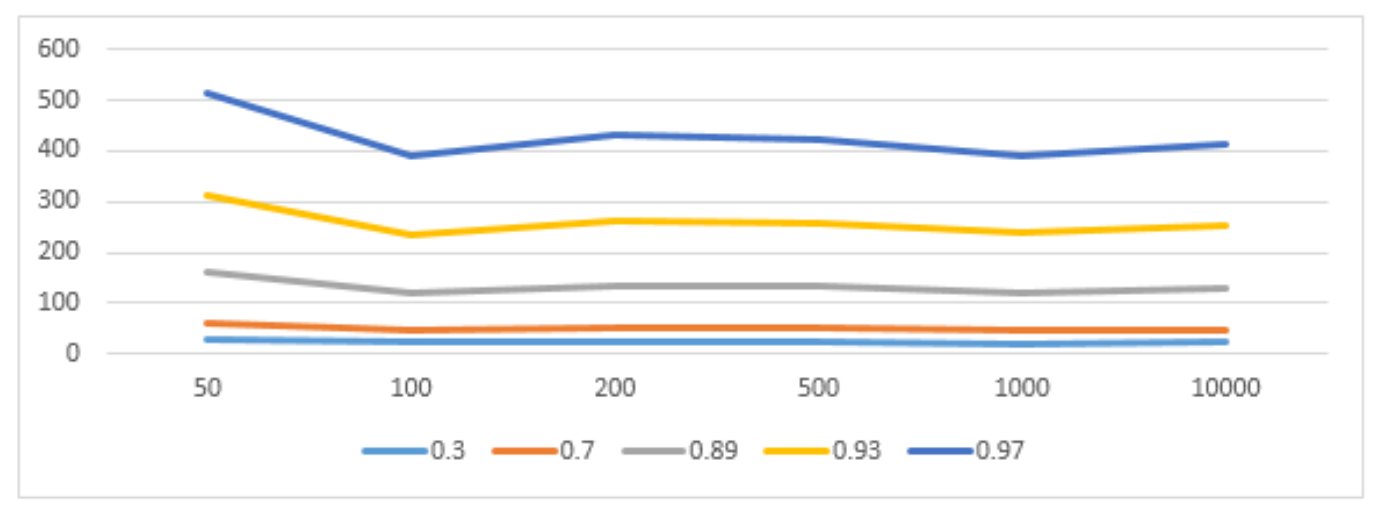

Figure2.3. Graph of Condition Number at different degree of correlation and sample size.

\section{CONCLUSION}

The adverse impact of ignoring multicollinearity on findings and data interpretation in regression analysis is very well documented in the statistical literature. The failure to identify and report multicollinearity could result in misleading interpretations of the results. Using the ratio of standardized regression slope to semi-partial correlation coefficient, this technique seem to be more sensitive to the multicollinearity among predictors in the model than the variance inflation factor and condition number methods. Although the ratio of standardized regression slope to semi-partial correlation coefficient method performs much better than variance inflation factor and condition number as an ill-conditioned model diagnostic, its performance is not entirely satisfactory. We therefore recommend using the ratio of standardized regression slope to semi-partial correlation coefficient method with a little caution about its sensitivity to severe collinearity.

\section{REFERENCES}

[1] Alin A. (2010). Multicollinearity. WIREs Computational Statistics. 2(3),Wiley Online Library.

[2] Weisberg, S. (1985). Applied Linear Regression. J. Wiley and Sons, NY.

[3] Naes, T. \& Martens, H. (1985). Comparison of prediction methods for multicollinear data. Communications in Statistics. Simulation and Computing, 14,545-576.

[4] Sambandam R.,(2003). Cluster Analysis Gets Complicated, the American Marketing Association ( Marketing Research,) Vol 15, No.1

[5] Farrar \& Glauber (1967), "Multicollinearity in regression analysis" review of economics and statistics, 49, pp. $92-107$

[6] Goldberger, A. S. (1991), A Course in Econometrics. Cambridge, MA: Harvard University Press.

[7] Greene, W. H. (2003). Econometric analysis. New Delhi: Pearson Education India

[8] Wooldridge, J. M. (2014). Introduction to econometrics: Europe, Middle East and Africa Edition. Boston: Cengage Learning.

[9] Hair, J., Anderson, R., Tatham, R.L., \& Black, W.C. (1998). Multivariate data analysis, (5th ed.), NJ: Upper Saddle River, Prentice-Hall.

[10] Wichers. R.C. (1975). The Detection of Multicollinearity: A Comment. The Review of Economics and Statistics, vol. 57, issue 3, 366-68.

[11] Huang, T. (1970). New Foraminiferida from the Taiwan Proceedings of the Geological Society of China. 13 : $108-114$

[12] Judge, George G., Griffiths, William E., Hill, Robin C., Lutkepohl, Helmut \& Lee, T.C., (1985), The Theory and Practice of Econometrics, New York: Wiley.

[13] Belsley,,D..A.,(1990), Conditioning Diagnostics: Collinearity and Weak Data in Regression, John Wiley, New York

[14] Kutner, M.H., Nachtsheim C.J., \& Neter,J., (2004). Applied Linear Regression Models. 4th Edn., McGraw Hill, New York.

[15] O'brien, R.M. (2007). A Caution Regarding Rules of Thumb for Variance Inflation Factors. Qual Quant 41, 673-690

[16] Freund, R.J. \& Wilson, W.J., (1998). Regression Analysis - Statistical modelling of a response variable. Academic Press, London. 444 pp. 
Performance of Ratio of Standardized Regression Slope to Semi-Partial Correlation in Identifying IllConditioned Model

[17] Hair J.F. Jr., Anderson R.E., Tatham R.L. Black W.C. (1995). Multivariate Data Analysis, 3rd edn. New York, Macmillan

[18] Mason, C.H. \& W.D. Perreault Jr.,(1991). Collinearity, power and interpretation of multiple regression analysis. J. Market. Res., 28: 268-280.

[19] Kim, R. S. (2011). Standardized regression coefficients as indices of effect sizes in metaanalysis. Unpublished doctoral dissertation, Florida State University.

[20] Peterson R.A. \& Brown,S.P. (2005), On the Use of Beta Coefficients in Meta-Analysis, Journal of Applied Psychology 90, 175-181.

[21] Aloe A.M. (2015), Inaccuracy of Regression Results I n Replacing Bivariate Correlation Research Synthesis Methods 6, $21-27$

[22] Aloe A.M. \& Becker B.J (2012) An effect of Regression predictors in Meta-analysis. Journal of Educational and Behavioral Statistics 37, $278-297$.

Citation: Ijomah Maxwell Azubuike \& Dozie Kelechi Celestine Nosike (2020). Performance of Ratio of Standardized Regression Slope to Semi-Partial Correlation in Identifying Ill-Conditioned Model. International Journal of Scientific and Innovative Mathematical Research (IJSIMR), 8(1), pp. 14-22. http://dx.doi.org/ 10.20431/2347 -3142.0801003

Copyright: (C) 2020 Authors, this is an open-access article distributed under the terms of the Creative Commons Attribution License, which permits unrestricted use, distribution, and reproduction in any medium, provided the original author and source are credited. 